\title{
High precision radial-velocity measurements of late-type evolved stars
}

\author{
I.N. Cummings, J.B. Hearnshaw, P.M. Kilmartin and A.C. Gilmore \\ Mt John University Observatory, Department of Physics and \\ Astronomy, University of Canterbury, Christchurch, New Zealand
}

\begin{abstract}
High dispersion spectra for 44 southern evolved stars of spectral type $\mathrm{K}$ or $\mathrm{M}$ have been obtained. From these observations relative radial velocities of $50 \mathrm{~m} / \mathrm{s}$ precision have been obtained by the method of digital cross-correlation. This method of achieving precise relative radial velocities for late-type stars, and the problems encountered in its use, are discussed. Using this method, statistically significant radial-velocity variations have been found. Two of the observed stars have their precise radial velocities presented and the potential mechanisms of their variation examined.
\end{abstract}

\section{Introduction}

Late-type evolved stars range across the upper right of the HR diagram, bounded by the variable red giants, like Mira and semi-regular variables, and the pulsating supergiant RV Tauri stars. In the past decade it has been discovered that stars at the center of this region of the diagram are not necessarily constant in photometry and radial velocity, as previously thought (see Walker et al. (1989)). This was the motivation for observations of $44 \mathrm{~K}$ and $M$ giants, bright giants and supergiants to be obtained, from November 1993 to February 1997, in the hope of helping to determine how common this phenomenon is, whether it is related to the variations seen for other stars in this region of the HR diagram and whether there is any subset of this region that can be regarded as containing mainly constant stars.

\section{Equipment}

The instrumentation used to obtain the radial velocities is illustrated schematically in Figure 1. The telescope is the 1-m McLellan reflector at the Mt John University Observatory. From there the light is taken to an echelle spectrograph by an optical fiber, to avoid flexure and guiding errors. The spectrograph is housed in an insulated cabinet which is kept in an air-conditioned room. The detector is a liquid nitrogen cooled Thomson CCD, with $384 \times 576$ pixels which are $23 \mu \mathrm{m}$ square in size. Observations are taken over orders $41-46$ and include the wavelengths seen in Table 1 . Observations of each star are immediately preceded and followed by two thorium exposures to allow interpolation of the dispersion to the mid-time of the stellar exposure. 


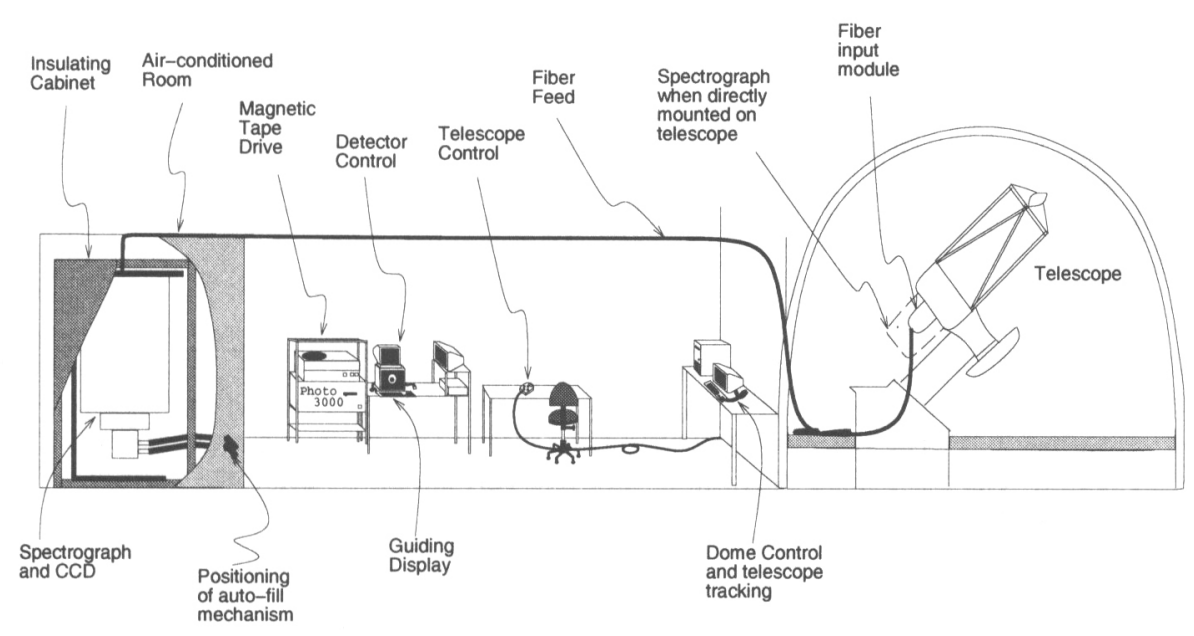

Figure 1. Schematic illustration of the layout for equipment used to obtain the spectroscopic observations

\begin{tabular}{|l|l|l|l|l|}
\hline Order & $\begin{array}{l}\lambda \text { Range } \\
(\AA)\end{array}$ & $\begin{array}{l}\lambda_{\mathrm{C}} \\
(\AA)\end{array}$ & $\begin{array}{l}\delta \lambda_{\mathrm{C}} \\
(\mathrm{m} \AA)\end{array}$ & $\begin{array}{l}P \\
(\AA / \mathrm{mm})\end{array}$ \\
\hline 41 & $5618-5643$ & 5630 & 191 & 2.09 \\
42 & $5484-5509$ & 5499 & 186 & 2.04 \\
43 & $5357-5381$ & 5369 & 182 & 2.00 \\
44 & $5235-5259$ & 5249 & 178 & 1.95 \\
45 & $5119-5142$ & 5132 & 174 & 1.91 \\
46 & $5008-5030$ & 5020 & 170 & 1.87 \\
\hline
\end{tabular}

Table 1. This table gives some of the vital statistics for the CCD over the wavelength range observed for this project where: $\lambda_{\mathrm{C}}$ is the wavelength in the center of the used part of the order, $\delta \lambda_{\mathrm{C}}$ is the resolution at $\lambda_{\mathrm{C}}$ and $P$ is the plate-factor.

\section{Reductions}

The reductions of these CCD images are for the most part standard, where Figaro is used to produce six 1-dimensional spectra for each exposure. An example is shown in the left-hand side of Figure 2 .

The second part of the reduction procedure is done by treating each order independently. The first step is to obtain dispersion solutions for the orders from the two sets of thorium spectra and average them. It is then possible to find the velocity shift between the stellar spectrum and some reference spectrum of the same star taken on a previous occasion by cross-correlating the two rebinned spectra. The cross-correlation function that results has data points at $2.4 \mathrm{~km} / \mathrm{s}$ intervals and this is fitted to find its peak, which gives the change in radial velocity for the star. For each exposure this is then averaged over the six orders. Using this method of reduction, relative radial velocities of about 

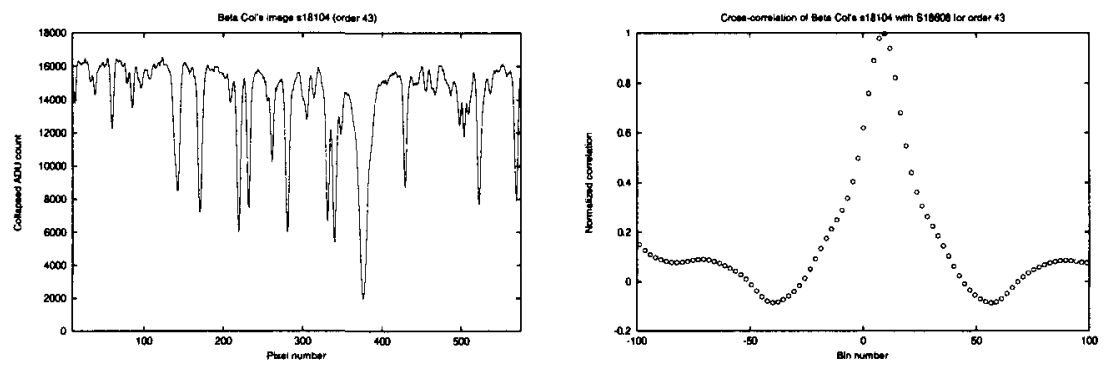

Figure 2. The left-hand diagram is the 43rd order of a typical K-type star, $\beta$ Col. The right-hand diagram is the cross-correlation function that results from two such spectra.

$50 \mathrm{~m} / \mathrm{s}$ precision have been obtained for the 44 stars observed. However, there are a few details on the cross-correlation function creation and fitting that need further discussion.

For well spread out lines a gaussian gives a good fit to the cross-correlation function. Unfortunately for late-type evolved stars the lines in the wavelength range observed are heavily blended. This produces the cross-correlation function shown in the right-hand side of Figure 2. These can be very non-gaussian and can not be fitted well by a single gaussian. This is improved by the use of a three-gaussian fit, where the three gaussians consist of a main central gaussian, with two smaller gaussians symmetrically distributed about it. This is because the distribution of points around the cross-correlation function peak is not symmetric and the fitting-function is not identical to the data points' distribution. This causes the least-squares fit to the cross-correlation function to be dragged to one or other side of the real peak position, depending on the distribution of the data points. Asymmetry will produce a similar error, although the symmetric three-gaussian fit will be less able to compensate for it.

\section{Results}

After about a year of observations, 13 stars had their observations discontinued in order to study a typical selection of stars more closely. In addition, 500 days after the first spectroscopic measurements were taken, 13 stars of the 31 stars still being observed began to have broad-band photometry taken. This was done because any one of orbital motion, rotation with starspots, pulsation and the movement of large convective cells can cause apparent radial-velocity variations and knowledge of the relationship between radial-velocity and light variation can help distinguish between them. In addition, the expected timescales for radial pulsation (predicted using Ostlie \& Cox (1986) and Schaller (1990)), rotational modulation and estimations of convective-cell life-times, were used to try and determine which mechanism was responsible for the observed variations. The typical range of timescales for these mechanisms is shown in Table 2.

All but eight of the 44 stars surveyed for this research have a less than $1 \%$ chance of being constant in radial velocity, where the initial observing list was 


\begin{tabular}{|c|c|c|c|c|}
\hline $\begin{array}{l}\text { Star } \\
\text { Type }\end{array}$ & $\begin{array}{l}P_{0} \\
\text { (days) }\end{array}$ & $\begin{array}{l}P_{\text {rot }} \\
\text { (days) }\end{array}$ & $\begin{array}{l}t_{\text {cell }} \\
\text { (days) }\end{array}$ & $\begin{array}{l}t_{\mathrm{obs}} \\
\text { (days) }\end{array}$ \\
\hline $\mathrm{K}$ giants & $1-50$ & $\geq 30-500$ & $15-250$ & $250-\gtrsim 2000$ \\
\hline$M$ giants & $16-70$ & $\geq 140-500$ & $70-240$ & $13-\gtrsim 1000$ \\
\hline supergiants & $\sim 100$ & $\gtrsim_{1000}$ & $\sim 400$ & $\sim 40 \gtrsim 1000$ \\
\hline
\end{tabular}

Table 2. Typical timescales for the 44 star observed for this project. $P_{0}$ is the predicted linear non-adiabatic radial-pulsation period; $P_{\text {rot }}$ is the period of rotation implied by a $v_{\text {equ }} \sin (i)$ of $20 \mathrm{~km} / \mathrm{s}$ (an estimated conservative upper limit); $t_{c e l l}$ is the estimated timescale for the movement of large convective cells, if the radial-velocity variations they produce have an amplitude of $100 \mathrm{~m} / \mathrm{s} ; t_{\mathrm{obs}}$ is the observed timescale in the relative radial velocity.

chosen with a preference for stars variable in light. These 36 stars are made up of: $16 \mathrm{M}$ giants, $15 \mathrm{~K}$ giants, $4 \mathrm{~K}$ supergiants, $1 \mathrm{M}$ supergiant.

For the significantly varying $\mathrm{K}$ giants the observed radial-velocity timescales are hundreds of days, which is not consistent with acoustic pulsation predictions. Hence the possible causes of the observed radial-velocity variation timescales are binary motion and rotational modulation, although non-acoustic non-radial pulsation can not be ruled out. There are four $\mathrm{K}$ giants which are certainly present in binaries and three or four which certainly have timescales due to rotational modulation. For the remaining $\mathrm{K}$ giants it is difficult to differentiate between rotational modulation and a binary orbit.

This is a general problem for long-term radial-velocity variations - even concurrent photometry can not fully differentiate the two, because although the same variation in photometry will rule out a wide binary orbit, no variation in photometry does not necessarily rule out a starpatch like that on $\xi$ Boo (Gray \& Toner (1988)). In addition, an erratic long-term radial-velocity variation may be due to the superposition of a wide binary orbit and another intrinsic variation of similar amplitude.

For $\mathrm{M}$ giants the situation is very different. Of the 16 significantly varying stars, 11 have timescales which must be due to acoustic pulsation. In addition to any short-term radial-velocity variations, most $M$ giants have a long-term radialvelocity variation, which could be due to a binary orbit or rotational modulation. The inability to distinguish between long-term radial-velocity variations due to a binary orbit and rotational modulation has already been discussed, but for the $\mathrm{M}$ giants this is made worse because convective-cell timescale estimations are also often comparable. This is because the amplitude of the $M$ giant variations is typically larger than those of $\mathrm{K}$ giants, which implies fewer and larger cells, which in turn implies longer timescales.

Like $M$ giants, the five supergiants observed for this research have both short and long-term timescales present in their radial velocities. For three out of five of these stars there is a timescale which can only correspond to acoustic pulsation and there is difficulty distinguishing between timescales due to binary orbits and surface features. 
For two fairly typical stars, precise radial velocities will now be presented and the potential mechanisms of their variation will be examined.

\subsection{A K giant}

The relative radial velocities in Figure 3 belong to the $\mathrm{K} 4$ giant, $\kappa$ Pyx. The only convincing timescale revealed by a periodogram of this data is of about 530 days. This timescale is not present in the photometry, and as can be seen (see the righthand side of Figure 3), visual inspection does not reveal any obvious correlation between the two.
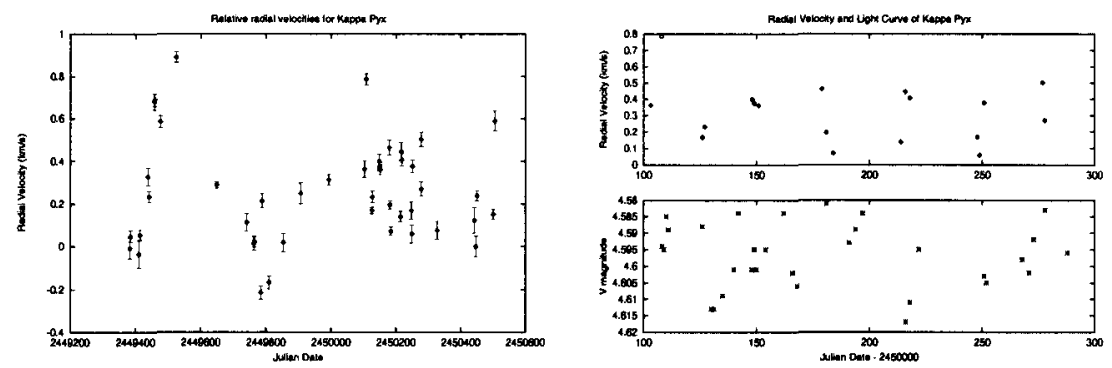

Figure 3. The relative radial velocity for $\kappa$ Pyx. A relatively well sampled region of concurrent radial velocity and visual luminosity is shown to the right.

The fundamental radial-pulsation prediction for $\kappa$ Pyx is about 10 days. Hence the observed radial-velocity timescale is far too long for an acoustic pulsation. In addition, the estimated convective-cell timescale is over a factor of two too short. However, the line broadening in this star is compatible with the value of $v_{\text {equ }}$ implied by this timescale, as is the rotation expected for $\mathrm{K}$ giants of a few hundred days or longer. It can not definitely be said that this timescale has arisen from rotation modulation, because it is often difficult to distinguish the effects of rotational modulation from binary orbits.

\subsection{An $M$ giant}

The relative radial velocities in Figure 4 belong to the M5 giant $\mathrm{L}^{2} \mathrm{Pup}$. It has both a long-term radial-velocity variation of about 435 days and a shorterterm radial-velocity variation of about 140 days. The latter is also present in its concurrent visual photometry and fitting a sine curve to both the concurrent data subsets seen in Figure 4, indicates that the two observables have radial velocity reaching minimum recessional velocity 29 days after minimum visual luminosity, which agrees well with visual inspection of the data sets.

The fundamental radial-pulsation prediction for $\mathrm{L}^{2} \mathrm{Pup}$ is 64 days. This is significantly shorter than the 140-day timescale, hence if it is due to an acoustic pulsation the star would have had to have suffered more mass loss then the evolution models assume, which is not unknown. Although the line broadening in this star is compatible with the value of $v_{\text {equ }}$ implied by either of these timescales, rotational modulation is unlikely to be the cause of the 140-day timescale because the star would have to be rotating much faster than is usual for late-type giants. 

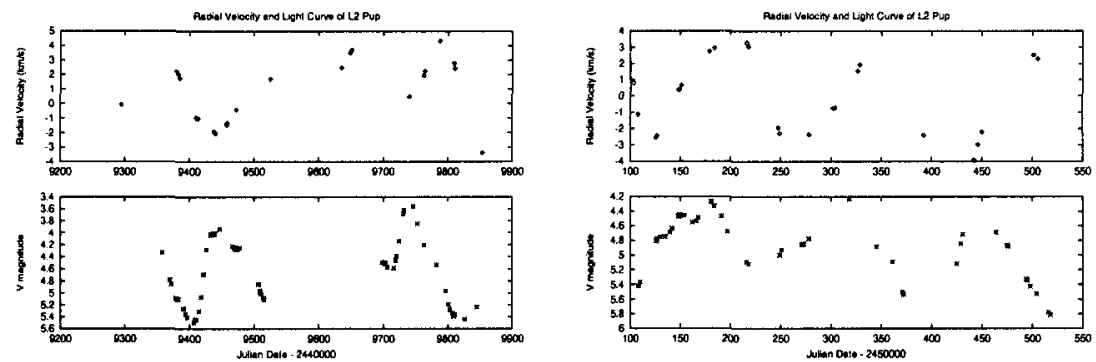

Figure 4. Two relatively well sampled regions of concurrent radial velocity and visual luminosity for $\mathrm{L}^{2}$ Pup.

It is also unlikely that the ascent and descent of large convective cells could cause these timescales because the 140-day timescale's amplitude is $2.5 \mathrm{~km} / \mathrm{s}$, which would require that only a few cells be present on the visible surface of the star, and the 435-day timescale is over a factor of two times smaller than the timescale estimated for convective cells.

A wide binary orbit would not be expected to give variations in light, so unless a non-acoustic non-radial pulsation is responsible, the 140-day timescale of this star must caused by some kind of acoustic pulsation and this star must have suffered unexpected mass loss.

Acknowledgments. We thank M. Clark for his help in obtaining some of the spectra. I.N.C. thanks the Wood Fund at the University of Canterbury and the Physics and Astronomy Department of the University of Canterbury for making funds available for participation in the conference. This paper is based on a thesis which has been submitted to the University of Canterbury in partial fulfilment of the requirements for a Doctor of Philosophy in Astronomy.

\section{References}

Gray, D.F. \& Toner, C.G. 1986, ApJ, 310, 277

Ostlie, D.A. \& Cox, A.N. 1986, ApJ, 311, 864

Schaller, G. 1990, in Confrontations Between Stellar Pulsation and Evolution (ASP Conf. Ser., 11), Cacciari, C. \& Clementini, G., San Francisco: Astron. Soc. Pacific, 300

Walker, G.A.H., Yang, S., Campbell, B., \& Irwin, A.W. 1989, ApJ, 343, L21

\section{Discussion}

Horner: I would like to reiterate something that Tim Brown said earlier today, i.e., that the treatment of the ends of the spectrum is very important when crosscorrelation is used. Effects at the ends of the spectrum can introduce errors on the order of 0.1 pixel shifts. I investigated this while I was a graduate student. 
You might find that you can reduce the rms noise in your velocities by using the least-squares method instead of cross-correlation.

Verschueren: A technical comment - the problems you mentioned regarding the fitting of the ccf are discretization errors. I refer to our paper in A\&AS, in 1995, that shows that one should always fit as close as possible to the top of the ccf, and use high-frequency Fourier filtering to reduce the random errors. All correct radial-velocity information is in the few pixels at the top of the ccf. The rest of the peak contains irrelevant information and the substructure destroys the result (for single-lined spectra of course).

Cummings: While problems due to line blending get worse the farther you go from the peak of the ccf, it is generally agreed that the bulk of the radial-velocity information is in the steep sides of lines near the line core, which would seem to indicate that this is also where the bulk of the radial-velocity information is in the ccf. The discretization error is worse at steeper slopes but both fitting with a curve that agrees better with the ccf data-point distribution, and having better pixel resolution, soon make the discretization error negligible. Hence it is still probably a better approach to fit down further on the ccf than the peak, and be aware of the discretization effect, than to throw away this valuable party of the ccf.

Larson: I congratulate you on attempting to explain the various sources for the short, long, and all other time frames for the periodicites seen in the $K$ and $M$ giants. Perhaps your work will help inspire the theorists to catch up with the observations. I look forward to reading your thesis.

Cummings: Yes, more and better models of these stars would be good. I was at the pulsation conference in Los Alamos last year, and after my talk several people made the same comment. Unfortunately all the theorists I talked to said, "No! Those stars are too hard! We want to do the nice simple early-type stars where progress comes easily."

Mazeh: I agree with the approach suggested by a previous comment, that one should interpolate the cross-correlation peak and not fit any shape to it.

Cummings: I would like to, but there are only three points near the peak of my ccf. 